\title{
The odd-even effect in multiplication: Parity rule or familiarity with even numbers?
}

\author{
ALIETTE LOCHY and XAVIER SERON \\ Université Catholique de Louvain, Louvain-La-Neuve, Belgium \\ MARGARETE DELAZER \\ Universitätklinik für Neurologie, Innsbruck, Austria \\ and \\ BRIAN BUTTERWORTH \\ University College of London, London, England
}

\begin{abstract}
This study questions the evidence that a parity rule is used during the verification of multiplication. Previous studies reported that products are rejected faster when they violate the expected parity, which was attributed to the use of a rule (Krueger, 1986; Lemaire \& Fayol, 1995). This experiment tested an alternative explanation of this effect: the familiarity hypothesis. Fifty subjects participated in a verification task with contrasting types of problems (even $\times$ even, odd $\times$ odd, mixed). Some aspects of our results constitute evidence against the use of the parity rule: False even answers were rejected slowly, even when the two operands were odd. We suggest that the odd-even effect in verification of multiplication could not be due to the use of the parity rule, but rather to a familiarity with even numbers (three quarters of products are indeed even).
\end{abstract}

Besides the issues concerning the representation format in which parity information is coded and the way this information is retrieved (Campbell \& Clark, 1992; Clark \& Campbell, 1991; Dehaene, Bossini, \& Giraux, 1993; McCloskey, Aliminosa, \& Sokol, 1991; McCloskey, Caramazza, \& Basili, 1985), the role of parity in arithmetic has also been studied (Krueger, 1986; Krueger \& Hallford, 1984; Lemaire \& Fayol, 1995; Lemaire \& Reder, 1999). In fact, several studies have proposed that subjects have access and sometimes use parity information without explicit awareness when doing mental calculation. For instance, it seems that in some circumstances, when subjects are verifying simple arithmetic operations (e.g., 2 $\times 3=6$ or $2+3=5$ ), they do not retrieve the result but use a shorthand strategy for checking for the plausibility of the proposed result. Among the cues used for checking that plausibility, its parity has proven to be relevant. It has been found that adults reject a false result more rapidly when its parity is incongruent with the parity of the correct answer (e.g., $2 \times 3=7)$ than when it is congruent with it (e.g., $2 \times 3=8$ ). This incongruence ef-

\footnotetext{
This work was supported by the Belgian National Scientific Research Funds (FNRS) and by the Belgian Federal government (I.U.A.P. on temporal control of dynamic tasks situations and the nature of knowledge representation). We thank Marie-Pascale Noël and Agnesa Pillon for their helpful comments on this work. Correspondence should be addressed to A. Lochy, Université Catholique de Louvain, Faculté de Psychologie, 10 place Cardinal Mercier, 1348 Louvain-La-Neuve, Belgium (e-mail: lochy@neco.ucl.ac.be).
}

fect has been observed for both addition (Krueger \& Hallford, 1984) and multiplication (Krueger, 1986), and it has been attributed to the use of a rule. In product verification, subjects likewise use the rule "the true product must be even if any multiplier is even; otherwise it must be odd" (Krueger, 1986). For Krueger, evenness is more salient than oddness since detecting a single even multiplier indicates that the product must be even, whereas all multipliers must be odd for the result to be odd. This author conceived the parity rule as a strategy that permits subjects to bypass a normal two-step verification process in which the subjects first retrieve the result in memory, and then compare it with the proposed one. These two processes, the parity rule checking one and the verification one (retrieval plus comparison), would be initiated in a parallel-race model.

This parallel-race interpretation was supported by the results of Lemaire and Fayol (1995). These authors speculated that if a parity rule checking process is going on in parallel with the retrieval of the correct answer, it should be more efficient early in the verification process and for difficult problems - that is, as long as the correct answer is not completely retrieved from memory. That is exactly what they found: In a product verification task, the oddeven effect was significant only for difficult problems and for the shortest delay between the presentation of the operands and the onset of the proposed answer.

Lemaire and Siegler (1995) found that second-grade children committed errors in multiplication that reflected the odd-even status of the target response. However, the 
pattern of errors first tended to correspond to the addition pattern of the rule, and changed over the school year ${ }^{1}$ toward the multiplication pattern. The parity effect thus seems to develop with practice and with the constitution of an integrated network of multiplication facts, consistent with the adaptive strategy choice model (ASCM; Siegler \& Lemaire, 1997; Siegler \& Shrager, 1994).

Furthermore, Lemaire and Reder (1999) found that the use of the parity rule depended on stimuli and contextual characteristics. They observed that when subjects were verifying complex problems $(49 \times 8=394)$, the incongruence effect was greater when both operands were even and when the efficiency of that strategy was high that is, when the proportion of problems violating the rule was high.

The results of these studies revealed a parity incongruence effect in product verification. However, they do not compel us to interpret the effect in terms of a parity rule. In this paper, we will propose an alternative interpretation: We will argue that this effect could arise from the distributional properties of the products. Within the multiplication tables, even numbers are three times more likely to be the result of multiplication than odd numbers, since three quarters of simple multiplication give an even result. On this basis, even numbers might seem more plausible than odd ones in product verification tasks. In other words, the incongruence effect might be, in fact, an evenness familiarity effect.

An "evenness familiarity effect" may indeed be confused with a "parity incongruence effect" since for at least $75 \%$ of the products both effects would result in a shortening of the reaction time (RT) when the subject is verifying a false incongruent answer. Indeed, for the even $X$ even $(E \times E)$, even $X$ odd $(E \times O)$, and odd $X$ even $(\mathrm{O} \times \mathrm{E})$ types of problems, an incorrect odd answer will be more quickly rejected than an even one, either by the application of a parity rule checking strategy (because of the violation of the odd-even congruence with the rule) or by a familiarity judgment because an odd answer looks less familiar than an even one. The crucial type of product that may allow one to disentangle the two interpretations is the odd $\times$ odd product $(\mathrm{O} \times \mathrm{O})$. For that type of problem only do the two interpretations diverge for the expected performance: If the parity congruence rule is used, presenting an incongruent even product would also result in a shortening of RT since it violates the expected parity. On the contrary, if the verification process is influenced by evenness familiarity, an even incorrect product would be less easy to reject because even results are the more likely products in multiplication.

The studies reviewed above did not assess the incongruence effect for each of the four product types, and thus they do not allow us to choose between the two interpretations. An exception is the Lemaire and Reder (1999) study, which compared types of problems and found a greater parity effect when both operands were even than when one operand was even, and a reverse, but not significant, effect on $\mathrm{O} \times \mathrm{O}$ problems. The authors proposed that noting evenness as a feature invoking the parity rule is twice as likely when there are two even operands; however, no explanation was proposed for the reverse trend noted for two-odd problems.

Our proposition for an evenness familiarity effect may explain the pattern of results obtained in Krueger's (1986; Krueger \& Hallford, 1984) studies. Since three quarters of the products proposed as incongruent answers are odd, they may be rejected faster because of their lower likelihood of being the results of multiplication. Moreover, some characteristics of the stimuli used in these studies might have reinforced the evenness familiarity effect by favoring even numbers as plausible. Indeed, the probability of being correct was much higher for even $(36.11 \%$ in Experiment 1$)$ than for odd ( $13.8 \%$ ) results, which were then probably more easily judged as false (a ratio of $1: 2.6){ }^{2}$

In summary, the source of the so-called incongruence effect observed in verification tasks is ambiguous: It could be due either to the application of a parity rule, which predicts the parity of the product given the parity of the operands, or to the natural asymmetry existing in the odd-even distribution of the products in simple multiplication tables. Moreover, this effect might also arise, in part, from the imbalance in the stimulus material presented to the subjects (a higher proportion of even answers were correct), since this kind of situational variation has been shown to influence performance (Lemaire \& Reder, 1999; Reder, 1988).

The aim of this study was to question further the existence of a parity rule ${ }^{3}$ and to contrast this hypothesis with the evenness familiarity hypothesis by focusing more closely on the different types of problems and each of their latency patterns. We wanted to use a verification task like that of the aforementioned authors while controlling the potential biases that might explain the facilitation effects found on incongruent splits.

Simple arithmetic problems were presented for verification. False results were either congruent or incongruent with the parity of the correct answers. In the design of this task, we carefully equated the proportion of odd and even products in our list by adding filler items in order to avoid any response bias due to the unequal distribution of odd and even products and their correctness.

In order to obtain evidence allowing us to disentangle the "parity rule hypothesis" and the "evenness familiarity hypothesis," we considered the results for three types of problems: $\mathrm{E} \times \mathrm{E}, \mathrm{O} \times \mathrm{O}$, and mixed $(\mathrm{E} \times \mathrm{O}$ and $\mathrm{O} \times$ E) problems. The two hypotheses predict different effects on these types of problems: If subjects use a rule of the type "the true product will be even if any multiplier is even; otherwise it must be odd (or, schematically: $E X$ $\mathrm{E}=\mathrm{E} ; \mathrm{E} \times \mathrm{O}=\mathrm{E} ; \mathrm{O} \times \mathrm{E}=\mathrm{E} ; \mathrm{O} \times \mathrm{O}=\mathrm{O}$ ), " then an incongruence effect (i.e., shorter RTs for incongruent answers) should be observed for each type of problem. However, if evenness is more salient than oddness (as suggested by Krueger, 1986, p. 142), this incongruence effect ought to be the largest when both multipliers are even, smaller when one is even and the other odd, and 
smallest when both are odd. If, on the contrary, a familiarity effect of the type "an even answer is more plausible as a result of a multiplication and an odd answer is more unfamiliar and less plausible," two specific predictions can be made. First, the so-called incongruence effect should be observed only for $\mathrm{E} \times \mathrm{E}$ and mixed problems because, in these cases only, the false incongruent answer is odd (i.e., looks less familiar). In the case of $\mathrm{O} \times \mathrm{O}$ problems, by contrast, this "incongruence effect" should be reversed; that is, the facilitation effects should be observed on congruent false answers since they are odd and look less familiar. For example, and contrary to the use of a rule, $7 \times 9=61$ would be rejected more quickly than $7 \times 9=62$. This would clearly mean that, even in the case of $O \times O$ problems, subjects expect even answers, which would then be rejected more slowly. Second, subjects should take longer to reject even answers, which should lead to higher error rates (i.e., to be erroneously accepted more often) than odd ones, whatever the problem type.

\section{METHOD}

\section{Subjects}

Fifty undergraduate students at the Universite Catholique de Louvain ( 16 males and 34 females) participated in this experiment to receive credit in an introductory course. Mean age was 19 years, 8 months (range: $18-23$ years).

\section{Stimuli}

The stimuli were multiplication problems presented in a standard form $(a \times b=c)$, where $a$ and $b$ were single-digit operands and $c$ a two-digit number; the true products ranged in size from 27 to 72 . The basic set of equations consisted of 12 multiplication problems (Table 1). Four equations were selected for each type of problem (even $\times$ even, odd $\times$ odd, mixed).

All the selected problems were difficult, according to Campbell's (Campbell, 1987a, Appendix B) difficulty index, since they seem to be more sensitive to the odd-even effect (Lemaire \& Fayol, 1995).

Table 1

Stimuli of the Verification Task

\begin{tabular}{|c|c|c|c|c|c|}
\hline \multirow[b]{3}{*}{ Type } & \multirow{3}{*}{$\frac{\text { True Answer }}{0}$} & \multicolumn{4}{|c|}{ False Answer } \\
\hline & & \multicolumn{4}{|c|}{ Split } \\
\hline & & $\overline{1}$ & 2 & 3 & 4 \\
\hline \multicolumn{6}{|l|}{$\mathrm{E} \times \mathrm{E}$} \\
\hline $8 \times 4$ & 32 & 33 & 34 & 29 & 38 \\
\hline $4 \times 8$ & 32 & 33 & 34 & 29 & 38 \\
\hline $8 \times 6$ & 48 & 47 & 46 & 51 & 44 \\
\hline $6 \times 8$ & 48 & 47 & 46 & 51 & 44 \\
\hline \multicolumn{6}{|l|}{$0 \times 0$} \\
\hline $9 \times 7$ & 63 & 62 & 61 & 66 & 59 \\
\hline $7 \times 9$ & 63 & 62 & 61 & 66 & 59 \\
\hline $3 \times 9$ & 27 & 26 & 29 & 22 & 23 \\
\hline $9 \times 3$ & 27 & 26 & 29 & 22 & 23 \\
\hline \multicolumn{6}{|l|}{ Mixed } \\
\hline $6 \times 9$ & 54 & 53 & 52 & 57 & 58 \\
\hline $9 \times 6$ & 54 & 53 & 52 & 57 & 58 \\
\hline $8 \times 9$ & 72 & 73 & 74 & 69 & 76 \\
\hline $9 \times 8$ & 72 & 73 & 74 & 69 & 76 \\
\hline
\end{tabular}

Note-Type of problems: $\mathrm{E} \times \mathrm{E}=$ even $\times$ even; $\mathrm{O} \times \mathrm{O}=$ odd $\times$ odd.
These 12 problems were presented eight times each, for a total of 96 regular trials. In half of the eight presentations, the proposed answer was the correct product of the two operands, and in the other half, problems were presented with a false answer. The distance between the correct and the false proposed answer (i.e., splits) was $\pm 1,2,3$, or 4 (e.g., $9 \times 7=62,61,66,59$ ). Incongruent answers were at splits of \pm 1 or 3 , and congruent ones were at splits of \pm 2 or 4 from the correct answers (Table 1). The direction of these splits was controlled to avoid potential biases: The sum of positive and negative splits was equal to zero in each split condition; six false answers were larger and six were smaller than the correct answer.

The problems were of three different types consisting of four equations each (half of them were complementary: $a \times b=c, b \times$ $a=c)$ : even $\times$ even $(\mathrm{E} \times \mathrm{E})$, odd $\times$ odd $(\mathrm{O} \times \mathrm{O})$, and mixed $(\mathrm{E}$ $\times \mathrm{O}$ and $\mathrm{O} \times \mathrm{E}$ ). This factor should allow us to determine whether the mathematical rule $(\mathrm{N} \times$ even $=$ even; odd $\times$ odd $=$ odd $)$ or evenness familiarity $[\mathbf{P}($ even product $)>\mathbf{P}($ odd product $)]$ was responsible for the incongruence effect.

False answers were chosen in order to avoid potential confusion. First, all the false answers were not plausible multiplication results, because table-related products might increase response latencies (Ashcraft, 1992; Zbrodoff \& Logan, 1990). Second, we avoided numbers that could represent operation errors. ${ }^{4}$

Filler items were introduced in order to make equal proportions of true even, false even, true odd, and false odd trials. These filler items were also simple multiplication problems, from the set of 100 pairs of the single digits 0 to 9 that were not used as experimental items. Since the 12 correct problems were presented four times, and since three quarters of them were even $(32 / 48)$, we added 16 filler odd correct problems. There were thus 64 correct equations. However, in order to respect the true/false proportion, we added 16 false items to the 48 experimental ones: Half of them were odd and half were even. Subjects thus had to answer to a set of 128 problems ( 32 of each true odd - even and false odd - even conditions), of which 96 were experimental trials. These problems were presented twice, in two successive sessions (for a total of 256 trials, of which 192 were experimental ones).

\section{Procedure}

The equations were presented horizontally in the center of a computer screen (twinhead 400), via MEL/2.0. Each trial began with a 500 -msec ready signal (a line of ${ }^{* * *}$ ) in the center of the screen. The equations were then displayed horizontally in the center of the screen. They were presented in the standard form $(a \times b=c)$. Digits and arithmetic symbols were separated by blank spaces equal to the width of one character. Each character was $0.70-\mathrm{cm}$ high $x$ $0.50-\mathrm{cm}$ wide. Each subject sat alone in a dark room, with the head located $60-80 \mathrm{~cm}$ from the display screen. The equation remained on the screen until the subject responded, and trials were separated by an interval of $2,500 \mathrm{msec}$.

Since the parity incongruence effect is maximum at short stimulus onset asynchronies (SOAs; Lemaire \& Fayol, 1995), we chose to present the proposed answer and the two operands simultaneously (i.e., at $0-\mathrm{msec} \mathrm{SOA}$ ). Subjects were asked to respond "true" or "false" as quickly and accurately as possible by pressing the appropriate key of the numeric keyboard, with the index finger of each hand. For half of the subjects, the "true" response was on the right side and the "false" response on the left side. The reverse instruction was given to the other half of the subjects.

All subjects were individually presented with the same 192 experimental problems ( 4 pairs of operands $\times 3$ types $[\mathrm{E} \times \mathrm{E}, \mathrm{O} \times$ $\mathrm{O}$, mixed] $\times 2$ answers [true, false] $\times 4$ levels of splits in the false equations $\times 2$ sessions), mixed with the 64 filler items. The problems were presented in a pseudo-random order. We took care to avoid repetition of the same problem or presentation of the correct answer for another equation (a minimum interval of four trials). The total 
of 256 trials ( 192 experimental and 64 filler items) was separated into eight blocks of 32 trials each, which allowed subjects to rest if they needed to; these trials were preceded by a practice set of six trials not included in the analysis.

The whole experiment lasted approximately $30 \mathrm{~min}$. Subjects did not receive any feedback on their accuracy or speed.

\section{RESULTS AND DISCUSSION}

\section{General Analysis: True Versus False}

In the first analysis, trials were classified as true or false, and size of split was disregarded. A 2 (sessions) $\times$ 3 (problem type: $\mathrm{E} \times \mathrm{E}, \mathrm{O} \times \mathrm{O}$, mixed) $\times 2$ (answer: true vs. false) analysis of variance (ANOVA) was performed on the subjects' mean correct RT and percentage of errors $\left(F_{1}\right)$, with repeated measures on the last three factors and Student Newman-Keuls comparisons. The corresponding $2 \times 3 \times 2$ ANOVAs were performed on the item means $\left(F_{2}\right)$, treating types as between-items variables and the other factors as within-items variables.

The results indicated that subjects answered faster during the second session than during the first one (Table 2), which could be explained by a simple effect of trainingthe subjects may have become more familiar with the task and the restricted range of problems $\left[F_{1}(1,49)=48.68\right.$, $M S_{\mathrm{e}}=321,892.37, p<.0001 ; F_{2}(1,9)=62.81, M S_{\mathrm{e}}=$ $22,675.19, p<.0001]$. This was also reflected in error rates, which tended to be smaller in the second than in the first session, although this trend was significant only in the items analysis $\left[F_{1}(1,49)=2.49, M S_{\mathrm{e}}=0.0056\right.$, $\left.p=0.2 ; F_{2}(1,9)=82.9, M S_{\mathrm{e}}=0.0002, p<.0001\right]$.

True equations were answered $123 \mathrm{msec}$ faster than false equations $\left[F_{1}(1,49)=21.50, M S_{\mathrm{e}}=93,238.08, p<\right.$ $\left..0001 ; F_{2}(1,9)=84.35, M S_{\mathrm{e}}=2,162.62, p<.0001\right]$, a classic finding presumably reflecting the fact that the true answers primed the retrieval of the correct product in the arithmetic facts network (Campbell, 1987b, 1991). The subjects more often rejected a correct answer than they accepted a false one $\left[F_{1}(1,49)=30.22, M S_{\mathrm{e}}=0.0056, p<\right.$ $\left..0001 ; F_{2}(1,9)=5.20, M S_{\mathrm{e}}=0.0027, p<.05\right]$. Such an effect on error rates was previously reported by Krueger (1986, Experiment 2), who proposed to account for it by assuming that "internal noise is more likely to make a correct proposed product seem false than to make an incorrect proposed product seem true" (Krueger, 1986, p. 143).

There was an interaction between answer and problem type $\left[F_{1}(2,98)=3.42, M S_{\mathrm{e}}=58,639.48, p<.03\right.$;

Table 2

Mean Correct Latencies (in Milliseconds),

Standard Deviations, and Percent of Errors (PE) for

True and False Answers by Problem Type in the Verification Task

\begin{tabular}{|c|c|c|c|c|c|c|c|c|c|}
\hline \multirow[b]{2}{*}{ Type } & \multicolumn{3}{|c|}{ True Answer } & \multicolumn{3}{|c|}{ False Answer } & \multicolumn{3}{|c|}{$M$} \\
\hline & RT & $S D$ & $\mathrm{PE}$ & RT & $S D$ & PE & RT & $S D$ & PE \\
\hline- & ( & & & & & 7.1 & 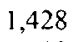 & 228 & 8.8 \\
\hline & & 2 & 10 & & 5 & 4. & & 26 & 7.6 \\
\hline $\mathrm{Mi}$ & 1,57 & 241 & 6. & 1,741 & 276 & 6.5 & 1,656 & 265 & 6.5 \\
\hline$M$ & 1,473 & 244 & 9.3 & 1,596 & 278 & 5.9 & 1,534 & 291 & 7.6 \\
\hline
\end{tabular}

$\left.F_{2}(2,9)=8.03, M S_{\mathrm{e}}=2,162.62, p<.01\right]$. When subjects were presented with a true answer, the $\mathrm{E} \times \mathrm{E}$ problems were solved faster than either the $\mathrm{O} \times \mathrm{O}$ or the mixed problems, but $\mathrm{RT}$ for the $\mathrm{O} \times \mathrm{O}$ and the mixed problems did not significantly differ from each other. With a false answer, however, the $\mathrm{E} \times \mathrm{E}$ and the $\mathrm{O} \times \mathrm{O}$ types did not significantly differ and were verified faster than the mixed problems (Student Newman-Keuls comparisons at al$\mathrm{pha}=.05$ ). There was only a trend for this interaction on error rates $\left[F_{1}(2,98)=11.80, M S_{\mathrm{e}}=0.0042, p<.0001\right.$; $\left.F_{2}(2,9)=0.0026, p=0.3\right]$ : No difference in function of answer was found for mixed problems, while for the $E X$ $\mathrm{E}$ and $\mathrm{O} \times \mathrm{O}$ types of problems, there were more errors on true answers than on false proposed answers. Since false answers can be either congruent or incongruent, we need to know what the effects are on congruency in order to interpret these interactions.

The mixed problems tended to be verified more slowly than the $\mathrm{O} \times \mathrm{O}$ problems, which tended to be verified more slowly than the $\mathrm{E} \times \mathrm{E}$ problems. However, this effect did not reach significance in the items analysis $\left[F_{1}(2,98)\right.$ $=20.62, M S_{\mathrm{e}}=126,411.82, p<.0001 ; F_{2}(2,9)=1.88$, $\left.M S_{\mathrm{e}}=112,103.03, p=.2\right]$. This insignificant trend might be due to some of the mixed problems being more difficult than the others 5 (e.g., $8 \times 9$ ).

Finally, there was a trend toward a triple interaction between problem type, session, and answer, but it did not reach significance in the subjects analysis $\left[F_{1}(2,94)=\right.$ 2.63, $\left.p=0.1 ; F_{2}(2,9)=4.56, M S_{\mathrm{e}}=1940.94, p<.04\right]$.

\section{Analysis Restricted to False Trials}

In order to study the so-called incongruence effect, and to contrast the parity rule and the evenness familiarity hypotheses, analyses were run on trials with a false proposed solution only. In the first analysis, false trials were classified according to their congruence with the parity of the correct answer. In the second one, they were classified according to the parity of the proposed answer per se (i.e., odd vs. even proposed answers).

Congruence of the proposed answer. Subjects' mean correct RTs and mean error rates to reject false trials were analyzed by a 2 (sessions) $\times 3$ (problem type: $\mathrm{E} \times \mathrm{E}, \mathrm{O} \times$ $\mathrm{O}$, mixed) $\times 2$ (congruence: congruent vs. incongruent) ANOVA, with repeated measures on the last three factors $\left(F_{1}\right)$; the corresponding $2 \times 3 \times 2$ ANOVAs were run on items mean correct RT and error rates, treating type as a between-items factor and sessions and congruence as within-items factors $\left(F_{2}\right)$

In regard to the effects of session and problem type, results revealed similar tendencies to those observed in the previous analysis. RTs were shorter $(-364 \mathrm{msec})$ during the second than the first session $\left[F_{1}(1,49)=40.20\right.$, $M S_{\mathrm{e}}=451,094.72, p<.0001 ; F_{2}(1,9)=65.15, M S_{\mathrm{e}}=$ $24,435.34, p<.0001]$, and a trend for an effect of type was observed in the subjects analysis on $\mathrm{RT}\left[F_{1}(2,98)=\right.$ 13.97, $M S_{\mathrm{e}}=204,089.82, p<.0001 ; F_{2}(2,9)=1.92$, $\left.M S_{\mathrm{e}}=132,405.26, p=.2\right]$ and on error rates $\left[F_{1}(2,98)=\right.$ 
Table 3

Mean Latencies (in Milliseconds ), Standard Deviations, and Percent of Errors (PE) for the Incongruent and Congruent False Proposed Answers by Problem Type in the Verification Task

\begin{tabular}{|c|c|c|c|c|c|c|c|c|c|}
\hline \multirow[b]{2}{*}{ Type } & \multicolumn{3}{|c|}{ Incongruent Answer } & \multicolumn{3}{|c|}{ Congruent Answer } & \multicolumn{3}{|c|}{$M$} \\
\hline & PT & $S D$ & $\mathrm{pF}$ & PT & ת & DI & & $S D$ & $\mathrm{E}$ \\
\hline 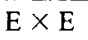 & & 4 & & & & & & 2 & 1 \\
\hline & & 306 & 4. & & & & & 308 & \\
\hline & & 227 & & & & & & 288 & \\
\hline 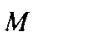 & 1,563 & 270 & 4.80 & 1,632 & 308 & 7.04 & 1,597 & 289 & 5.9 \\
\hline
\end{tabular}

$7.26, M S_{\mathrm{e}}=0.0059, p<.001 ; F_{2}(2,9)=4.61, M S_{\mathrm{e}}=$ $0.0007, p<.05$; see Table 3].

Incongruent answers were rejected faster $(-69 \mathrm{msec})$ than congruent ones $\left[F_{1}(1,49)=11.14, M S_{\mathrm{e}}=64,703.49\right.$, $\left.p<.001 ; F_{2}(1,9)=19.94, M S_{\mathrm{e}}=2,882.12, p<.001\right]$, and more errors were committed on false congruent $(7.04 \%)$ than false incongruent answers $\left[4.8 \% ; F_{1}(1,49)=10.78\right.$, $M S_{\mathrm{e}}=0.0065, p<.001 ; F_{2}(1,9)=9.54, M S_{\mathrm{e}}=0.00059$, $p<.01]$. However, this incongruence effect significantly interacted with problem type on RT $\left[F_{1}(2,98)=16.02\right.$, $M S_{\mathrm{e}}=58,955.50, p<.0001 ; F_{2}(2,9)=29.95, M S_{\mathrm{e}}=$ $2,882.12, p<.0001]$. Contrasts revealed that incongruent answers were rejected significantly more quickly than congruent ones for both the $\mathrm{E} \times \mathrm{E}$ problems $[-156 \mathrm{msec}$; $F_{1}(1,98)=10.31, M S_{\mathrm{e}}=58,955.50, p<.001 ; F_{2}(1,9)=$ $\left.50.66, M S_{\mathrm{e}}=2,882.12, p<.0001\right]$ and the mixed problems $\left[-152 \mathrm{msec} ; F_{\mathrm{l}}(1,98)=9.797, M S_{\mathrm{e}}=58,955.50\right.$, $\left.p<.01 ; F_{2}(1,9)=48.09, M S_{\mathrm{e}}=2,882.12, p<.0001\right]$. However, the opposite pattern was observed for the $\mathrm{O} \times$ $O$ problems: In these cases, incongruent answers were rejected significantly more slowly $(+100 \mathrm{msec})$ than congruent ones $\left[F_{1}(1,98)=4.24, M S_{\mathrm{e}}=58,955.50, p<.05\right.$; $\left.F_{2}(1,9)=20.81, M S_{\mathrm{e}}=2,882.12, p<.001\right]$. On error rates, a significant difference between congruent and incongruent answers was found only for the $E \times E$ type of problems: Congruent answers were more often erroneously accepted $(10.25 \%)$ than incongruent ones $(4 \%)$. Such a large difference did not appear for the $\mathrm{O} \times \mathrm{O}$ or the mixed problems $\left[F_{1}(2,98)=10.38, M S_{\mathrm{e}}=0.0061\right.$, $\left.p<.0001 ; F_{2}(2,9)=8.52, M S_{\mathrm{e}}=0.0005, p<.008\right]$.

There was a significant session $\times$ problem type $\times$ congruence three-way interaction on RT $\left[F_{1}(2,94)=7.4\right.$, $M S_{\mathrm{e}}=53,106.88, p<.001 ; F_{2}(2,9)=4.8, M S_{\mathrm{e}}=$ $6,715.61, p<.04]$. In Session 1, the incongruence effect was larger for the mixed $(-264 \mathrm{msec})$ than for the $\mathrm{E} X$ $\mathrm{E}(-89 \mathrm{msec})$ problems, whereas the opposite pattern appeared in Session 2 (i.e., a difference of $-41 \mathrm{msec}$ for mixed, and of $-222 \mathrm{msec}$ for $\mathrm{E} \times \mathrm{E}$ problems). No differences were noted between Sessions 1 and 2 for the $\mathrm{O} \times \mathrm{O}$ problems: Incongruent false answers were rejected more slowly than congruent ones in both sessions ( 98 and $102 \mathrm{msec}$ ). While this last finding is in disagreement with the use of the parity rule and favors the evenness familiarity hypothesis, the variations of the effect from the first to the second session for the $\mathrm{E} \times \mathrm{E}$ and the mixed problems might suggest that some kind of "degraded" rule, at least on the $\mathrm{E} \times \mathrm{E}$ problems, may well have been reinforced during the course of the task.

A trend for an interaction between problem type and session was found in the items analysis only on error rates $\left[F_{1}(2,48)=1.96, M S_{\mathrm{e}}=58,955.5, p=.2 ; F_{2}(2,9)=\right.$ $\left.5.26, M S_{\mathrm{e}}=0.00014, p<.04\right]:$ While the decrease in error rate between the first and the second sessions was about $5 \%$ for $\mathrm{E} \times \mathrm{E}$ and $\mathrm{O} \times \mathrm{O}$ types, it was only $2.3 \%$ on the mixed problems.

Parity of the proposed answer. In order to test the hypothesis that even answers should be rejected more slowly than odd ones, whatever the type of problem, ANOVAs with sessions (2), problem type (3: $\mathrm{E} \times \mathrm{E}, \mathrm{O}$ $\times O$, mixed), and parity of the false answer (2: odd vs. even) were performed on RTs and error rates for false problems correctly rejected, using both subjects $\left(F_{1}\right)$ and items means $\left(F_{2}\right)$. In the subject analysis, all factors were within-subjects factors, while in the items analysis, problem type was a between-items factor.

These analyses revealed that even answers were rejected significantly more slowly $(121 \mathrm{msec})$ than odd ones $\left[F_{1}(1,49)=45.16, M S_{\mathrm{e}}=55,028.28, p<.0001\right.$; $\left.F_{2}(1,9)=77.17 ; M S_{\mathrm{e}}=2,882.12, p<.0001\right]$, and that this evenness effect did not significantly interact with problem type [type $\times$ parity, $F_{1}<1 ; F_{2}(2,9)=1.33$, $\left.M S_{\mathrm{e}}=2,882.12, p=.3\right]$. The other significant effects revealed by these analyses were similar to those observed when analyses were done with the congruence factor rather than the parity one. The evenness familiarity prediction is thus confirmed by this analysis, despite a potential size effect going against the predicted trend: Even answers were of a smaller magnitude (mean size $=42.9$ ) than odd answers (mean size $=52.1)^{6}$

A similar pattern emerged from the error analyses: Even answers gave rise to more errors $(7.08 \%)$ than odd answers $\left[4.8 \% ; F_{1}(1,49)=9.35, M S_{\mathrm{e}}=.0081, p>.004\right.$; $\left.F_{2}(1,9)=10.29, M S_{\mathrm{e}}=.00059, p<.01\right]$, and this was especially apparent for the $\mathrm{E} \times \mathrm{E}$ problems $[10.25 \%$ of errors for even false answers against $4 \%$ for odd false answers; type $\times$ parity, $F_{1}(2,98)=11.43, M S_{\mathrm{e}}=.0052$, $\left.p<.0001 ; F_{2}(1,9)=8.14, M S_{\mathrm{e}}=.00059, p<.009\right]$. The contrasts revealed that the effect was significant for $\mathrm{E} X$ E problems $\left[F_{1}(1,98)=18.78, M S_{\mathrm{e}}=.0052, p<.0001\right.$; $\left.F_{2}(1,9)=39.73, M S_{\mathrm{e}}=.00059, p<.0001\right]$, while it did not reach significance for the mixed problems $\left[F_{1}(1,98)<\right.$ $\left.1 ; F_{2}(1,9)<1\right]$ or the $\mathrm{O} \times \mathrm{O}$ problems $\left[F_{1}(1,98<1\right.$; $\left.F_{2}(1,9)<1\right]$.

Discussion. If a parity rule was used to verify the equations, all the incongruent answers should have been rejected faster than congruent answers. This is not what we obtained: The incongruent false answers were rejected faster for the $E \times E$ and the mixed types of problems, while on the contrary, they were rejected more slowly for the $\mathrm{O} \times \mathrm{O}$ problems. When RTs were averaged on all problem types, a general incongruence effect appeared just because there are more $\mathrm{E} \times \mathrm{E}$ and mixed problems (two thirds) than $\mathrm{O} \times \mathrm{O}$ ones (one third), as in earlier studies (Krueger, 1986; Lemaire \& Fayol, 1995). Indeed, 
in $\mathrm{E} \times \mathrm{E}$ and mixed problems, congruent-incongruent answers are confounded with even-odd answers.

These results strongly indicate that faster rejection of false answers in a product verification task are not so much due to the incongruence of the false answer (even if it shortened RTs by $69 \mathrm{msec}$ when averaged over all types of problems), but to the fact that the answer is odd (latencies are shortened by $121 \mathrm{msec}$ in that case). We thus conclude that it is not the use of the parity rule that facilitates rejection of false incongruent answers, but the use of another kind of arithmetic knowledge, the higher proportion of even products in the multiplication tables.

This evenness effect was larger when both the operands and the proposed answer were even, on RTs as well as on error rate. This result was also found by Lemaire and Reder (1999), who suggested that "noting evenness as a feature that will invoke the parity rule is twice as likely when there are two even operands." This might also arise from a greater association of two even terms and an even answer, maybe in part due to the addition pattern of parity. The $E \times E$ type is the only one for which the parity of the answer is the same for both operations $(E \times E=E ; E+E=E$ ), while for the other types, the parity of the answer is different from that in addition $(\mathrm{E} \times \mathrm{O}=\mathrm{E}$ and $\mathrm{E}+\mathrm{O}=\mathrm{O} ; \mathrm{O} \times \mathrm{O}=\mathrm{O}$ and $\mathrm{O}+\mathrm{O}=$ E). This effect grew from the first to the second session; it suggests that exposure to the task may have reinforced this strong association, or that some subjects relied on some kind of rule for the $E \times E=E$ type of problem.

\section{GENERAL DISCUSSION}

This study's main purpose was to determine further the role of parity during verification of simple multiplication and to test an alternative interpretation to the parity rule checking strategy. We postulated that the so-called incongruence effect is not the result of a parity checking process based on a rule, linking the parity of the multiplicands to the parity of the product, but that it could be more adequately interpreted as an evenness familiarity effect. We proposed that an even number is considered as a more plausible correct product than an odd one because of the natural distribution of odd $(25 \%)$ and even $(75 \%)$ products in the multiplication tables. Our results support this interpretation since in a verification task, rejection times were longer for false even answers whatever the problem type. It is worth noting that when analysis was done on congruence, we replicated the finding of the previous studies: Incongruent answers were rejected with a 69-msec advantage relative to congruent ones. However, this was probably an artifact since it was only true when the analysis was carried out on all types of problems merged. Indeed, when detailing the different types of problems $(\mathrm{E} \times \mathrm{E}, \mathrm{O} \times \mathrm{O}$, mixed), we found an interaction between congruence and types of problem that revealed an interference of even answers. They were the most slowly rejected, and they corresponded to incongruent answers for $\mathrm{O} \times \mathrm{O}$ problems and to congruent ones for the other two types of problems. Such a result is not compatible with the parity rule since in that theoretical perspective, rejection times should have been faster for false incongruent answers (i.e., even ones) in the case of $\mathrm{O} \times \mathrm{O}$ problems.

The replacement of the "congruence" variable by the "parity" of the proposed answer confirmed that the familiarity with even answers in multiplication influences the plausibility process. Even answers were rejected 121 msec more slowly than odd ones, and this effect was similar for all types of problem. However, subjects were more prone to accept false even answers when proposed as answers to $\mathrm{E} \times \mathrm{E}$ type problems: This shows a greater effect of the plausibility of even answers in that case. This might be due to the fact that it is the only type of problem for which the parity status of the answer is the same in addition $(E+E=E)$, which may reinforce the association with an even correct answer. Another interpretation of this effect is proposed by Lemaire and Reder (1999), who suggested that subjects are biased in their strategy selection by both stimuli and contextual characteristics. The situation presented to subjects in the present experiment was a "neutral" one and did not favor any strategy to be used (the probability to see an even or an odd correct or false proposed answer was the same). On the other hand, stimuli characteristics such as the number of even operands influenced the effect: These authors suggested that problems with two even operands are twice as likely to invoke the evenness feature of the rule. However, even if the familiarity effect was stronger for $\mathrm{E} \times \mathrm{E}$ problems, it was the reverse for $\mathrm{O} \times \mathrm{O}$ problems, which is not explainable by the influence of the parity rule.

Our interpretation does not rule out the particular processes involved in such a plausibility checking strategy (parallel-race competing processes; Krueger, 1986; Lemaire \& Fayol, 1995), but only the content of that plausibility strategy. Instead of the parity rule $(E \times E=$ $\mathrm{E}, \mathrm{E} \times \mathrm{O}=\mathrm{E}, \mathrm{O} \times \mathrm{E}=\mathrm{E}, \mathrm{O} \times \mathrm{O}=\mathrm{O}$ ), it could be formulated as an implicit known probability of the type $[\mathrm{P}($ correct product being even $)>\mathrm{P}$ (correct product being odd)]. Thus, the familiarity effect could account for the effects found previously, like the difficulty effect. As Lemaire and Fayol (1995) suggested, when the correct answer is retrieved in memory, as for easy problems, the subject's decision is based on that retrieved answer rather than on the plausibility process. The latter would help decision making as long as the correct answer is not completely retrieved, as when difficult problems are involved. In our experiment, even if we did not manipulate the difficulty level of the problems, we realized afterward that mixed problems were larger in size and thus were more difficult than the other types: Indeed, the parity effects were larger for those difficult problems. Another effect that may be accounted for by our interpretation is the age-related effect. Lemaire and Siegler (1995) found that the error patterns of second-grade children emerged during the course of the school year: They first corresponded to the addition pattern and then grew to 
correspond to the multiplication pattern. This may be understood as reflecting the constitution of the arithmetical fact network in multiplication, and the coding of parity inside that network.

A third effect that we replicated (Lemaire \& Reder, 1999 ) is a change over the course of the experiment: We indeed found interactions with the factor session involved. More specifically, the effect was reinforced on $\mathrm{E} \times \mathrm{E}$ problems on error rate. This means that subjects associated more strongly an even answer with an $\mathrm{E} \times \mathrm{E}$ problem in the second part of the experiment, and thus that they learned the rule for this type of problem. However, the reverse incongruence effect on $\mathrm{O} \times \mathrm{O}$ was the same over both sessions, and opposite to the parity rule perspective. Thus, the familiarity hypothesis has to integrate some modulations due to learning effects: Even answers are more plausible in general, but it is more the case when the two operands are even and when there is some practice.

One could argue that the familiarity hypothesis could account for the parity effects found in multiplication only, but not for those in addition, since there is no bias toward even numbers in the distribution of results in addition. Indeed, $\mathrm{E}+\mathrm{E}=\mathrm{E}, \mathrm{O}+\mathrm{O}=\mathrm{E}, \mathrm{E}+\mathrm{O}=\mathrm{O}$, and $\mathrm{O}+\mathrm{E}=\mathrm{O}$ : There are thus $50 \%$ even answers and $50 \%$ odd answers. Thus if the familiarity toward even numbers is strictly linked to the network configuration, no difference is expected between odd and even answers. But even numbers could also be more familiar in general, not only because of their higher proportion in multiplication, but because they would be processed more often in arithmetical tasks (e.g., 9 out of the 15 possible combinations of odd and even numbers across the four operations have an even result $[\mathrm{E}+\mathrm{E}=\mathrm{E} ; \mathrm{E} / \mathrm{O}=\mathrm{E} \ldots]$; we are more used to counting by two with even than with odd numbers, etc.). Thus the evenness familiarity hypothesis is either strictly limited to each operation or a very general property of arithmetic and numbers.

The effect of a parity rule on addition has never been replicated since the original experiments of Krueger and Hallford (1984), where it was found in one of two experiments. Moreover, biases in the item selection could influence the interpretation of the results (e.g., sums with one of the addends equal to 0 or $1-$ no need to retrieve a result; sums equal to 10 , or ties-very easy; etc.). Furthermore, a close examination of the pattern of results reveals that it is not so much " $2+2=5$ " that looks strange, but " $2+2=7$." Indeed, the only downward dip is from split 2 to split 3 . The authors interpreted their result by the conjoint action of the parity rule and of the split effect, which go in the same direction only from split 2 to split 3 , and thus reinforce each other, whereas they are opposed at the other splits.

In terms of the congruent-incongruent answers, if the parity rule and the split have additive effects, as suggested by Krueger and Hallford (1984), there should be no difference at all between incongruent and congruent answers (split $1=$ split 2 ; split $3=$ split 4 ; thus the mean of split $1+$ split 3 would equal the mean of split $2+$ split 4 ). If the (general) familiarity with even numbers and the split effects are added, there should be a slight difference favoring the rejection of congruent answers. Indeed, the split effect is a linear decrease in RT, while the parity effect would not manifest when all problems are averaged (since there are $50 \%$ of each): Thus the addition of these effects would give rise to a pattern of results in which there is a slight decrease from split 1 to split 4 , and the average of the congruent versus incongruent answers would result in slower rejection times for incongruent (mean distance $=2$ ) than congruent (mean distance $=3$ ) ones. Indeed, this is what Krueger and Hallford (1984) obtained: The mean rejection time incongruent answers was $1,375 \mathrm{msec}$, while that for congruent answers was slightly slower, with $1,362 \mathrm{msec}$. Thus, it seems that the results obtained by Krueger and Hallford could be explained by an alternative hypothesis of a general evenness familiarity. Further experiments are needed to test the clear predictions raised by these different hypotheses in relation to parity and addition, as well as to assess the more general familiarity with even numbers in different tasks requiring number processing.

The use of plausibility strategies based on stimuli characteristics has been widely reported in various domains (Ashcraft \& Battaglia, 1978; Lemaire \& Reder, 1999; Reder, 1982, 1988; Siegler, 1987, 1988; Siegler \& Lemaire, 1997; Siegler \& Shrager, 1994). In arithmetic, semantic information about numbers such as parity or magnitude has been shown to become quickly available during the processing of a stimulus and to influence subjects' decisions (Krueger, 1986; Lemaire \& Fayol, 1995; Lemaire \& Reder, 1999). What has been clearly established is a sensitivity of subjects toward regularities. In regard to parity, the subject of the present paper, our view differs from earlier ones in that we propose a familiarity effect that corresponds to a very global regularity pattern in multiplication: The proportion of even numbers is higher, and they are thus are more plausible. Earlier authors considered a more subtle sensitivity, linking the parity of the operands and results (Krueger, 1986; Lemaire \& Fayol, 1995; Lemaire \& Reder, 1999). Our data may be explained by the evenness familiarity perspective, except for the largest effect we observed on $\mathrm{E} \times \mathrm{E}$ problems. This could perhaps be due to a reinforcement of the association between even terms and even answers because of the addition pattern of the rule. Or, the sensitivity linking operands and answer is greater in this case, because evenness is a salient feature, as suggested by Krueger and Lemaire and Reder. What our data show is actually a kind of "degraded rule": The plausibility of even numbers seems to be greater than odd ones in general, and contrary to the mathematical rule in the case of $\mathrm{O} \times \mathrm{O}$. But it is also greater for $\mathrm{E} \times \mathrm{E}$ than for the other types. The level of expertise of subjects is certainly a variable that should be taken into account and studied further. Indeed, expert subjects, who process multiplication problems very often, would probably be more sensitive to the exact association between operands and answers. This view corresponds to Siegler' s ASCM (Lemaire \& Siegler, 1995; Siegler \& Shrager, 1994), where the parity infor- 
mation could be viewed as coded first at a global level, characterizing multiplication in general as generating even answers in most of the cases, and then at a more specific level, coding the parity of each of the operands and of the corresponding answer as a particular feature of each problem.

In conclusion, the present experiment obtained data that are not compatible with the use of the parity rule as such in verification of multiplication, since even false answers are more difficult to reject than odd ones. Our alternative explanation proposes an evenness familiarity effect that could be due to the higher proportion of even numbers in the multiplication tables, but perhaps also to their more frequent processing in daily life. Our findings reinforce the idea that semantic features of problems influence subjects' performance in arithmetic, and it has to be taken into account by the various models of numerical cognition.

\section{REFERENCES}

AshCRAFT, M. H. (1992). Cognitive arithmetic: A review of data and theory. Cognition, 44, 75-106.

Ashcraft, M. H., \& Battaglia, J. (1978). Evidence for retrieval and decision processes in mental addition. Journal of Experimental Psychology: Human Learning \& Memory, 4, 527-538.

CAMPBELL, J. I. D. ( 1987a). Network interference and mental multiplication. Journal of Experimental Psychology: Learning, Memory, \& Cognition, 13, 109-123.

CAMPBELJ., J. I. D. (1987b). Production, verification, and priming of multiplication facts. Memory \& Cognition, 15, 349-364.

CAMPBELL, J. 1. D. (1991). Conditions of error priming in number-fact retrieval. Memory \& Cognition, 19, 197-209.

CAMPBell, J. I. D., \& ClaRK, J. M. (1992). Cognitive number processing: An encoding-complex perspective. In J. I. D. Campbell (Ed.), The nature and origins of mathematical skills (pp. 457-491). Amsterdam: Elsevier.

Clark, J. M., \& Campbell, J. I. D. (1991). Integrated versus modular theories of number skills and acalculia. Brain \& Cognition, 17, 204-239.

Dehaene, S., Bossinı, S., \& Giraux, P. (1993). The mental representation of parity and number magnitude. Journal of Experimental Psychology: General, 122, 371-396.

KRUEGer, L. E. (1986). Why $2 \times 2=5$ looks so wrong: On the oddeven rule in product verification. Memory \& Cognition, 14, 141-149.

Krueger, L. E., \& Hallford, E. W. (1984). Why $2+2=5$ looks so wrong: On the odd-even rule in sum verification. Memory \& Cognition, 12, 171-180.

LEMAIRE, P., \& FAYOL, M. (1995). When plausibility judgments supersede fact retrieval: The example of the odd-even effect in product verification. Mernory \& Cognition, 23, 34-48.

Lemaire, P., \& Reder, L. M. (1999). What affects strategy selection in arithmetic? The example of parity and five effects on product verification. Memory \& Cognition, 27, 369-382.

LEMAIRE. P., \& SiEgLER, R. S. (1995). Four aspects of strategic change: Contributions to children's learning of multiplication. Journal of Experimental Psychology: General, 124, 83-97.

McCloskey, M., Aliminosa, D., \& SoKol, S. M. (1991). Facts, rules, and procedures in normal calculation: Evidence from multiple singlepatient studies of impaired arithmetic fact retrieval. Brain \& Cognition, 17, 154-203.
McCloskey, M., Caramazza, A., \& Basili, A. (1985). Cognitive mechanisms in normal and impaired number processing and calculation: Evidence from dyscalculia. Brain \& Cognition, 4, 171-196.

REDER, L. M. (1982). Plausibility judgment versus fact retrieval: Alternative strategies for sentence verification. Psychological Review, 89, 250-280.

REDER, L. M. (1988). Strategic control of retrieval strategies. In G. H. Bower (Ed.), The psychology of learning and motivation (Vol. 22, pp. 227-259). New York: Academic Press.

SIEGLER, R. S. (1987). The perils of averaging data over strategies: An example from children's addition. Journal of Experimental Psychology: General, 106, 250-264.

SiEGLER, R. S. (1988). Strategy choice procedures and the development of multiplication skills. Journal of Experimental Psychology: General, $117,258-275$.

SiEgLER, R. S., \& LemaiRe, P. (1997). Older and younger adults' strategy choices in multiplication: Testing predictions of ASCM via the choice/no choice method. Journal of Experimental Psychology: General, 126, 71-92.

Siegler, R. S., \& Shrager, J. (1994). Strategy choices in addition and subtraction: How do children know what to do? In C. Sophian (Ed.), Origins of cognitive skills (pp. 229-294). Hillsdale, NJ: Erlbaum.

ZBRoDOFF, N. J., \& LOGAN, G. D. (1990). On the relation between production and verification tasks in the psychology of simple arithmetic. Journal of Experimental Psychology: Learning, Memory, \& Cognition, 16, 83-97.

\section{NOTES}

1. Errors corresponding to the addition pattern fell from $81 \%$ to $60 \%$ between January and June, while for the multiplication pattern, they grew from $39 \%$ to $66 \%$.

2. Lemaire and Fayol's (1995) experiments presented the same kind of stimuli imbalance.

3. Originally, this study examined another question concerning the explicit knowledge of a parity rule, but we do not report the results here since it did not show any clear effect. Between the two sessions of the verification task, half of the subjects were asked to predict the odd-even status of the result of multiplication problems, where the operands of the problems were presented one after the other. The other half of the subjects had to perform a comparison task. However, these groups did not differ significantly in the analysis of the subsequent verification task. We thus decided to examine solely the effects of parity in the verification task.

4. To respect these restrictions, a few exceptions had to be introduced: (1) To avoid table-related products, the sum of splits is not equal to zero on split 1: 4 products are larger, and 8 are smaller than the correct results. (2) To avoid numbers that could represent operand errors, two problems are presented at nonhomogeneous splits, but respecting the congruence of the split condition. We included a false answer at -5 in split $3(9 \times 3=22)$, and a false answer at +6 in split $4(8 \times 4=38)$. The answers that we might propose at \pm 3 or \pm 4 were numbers of the 3 table and 4-table, respectively, which could generate a table-related interference effect.

5. Indeed, even if all the selected problems were classified as difficult (Campbell, 1987a, Appendix B), they were not homogeneous in size. The mean size of the correct products was 40 for the $E \times E$ problems, 45 for the $\mathrm{O} \times \mathrm{O}$, and 63 for the mixed.

6. We thank Patrick Lemaire for this remark.

(Manuscript received April 15, 1998; revision accepted for publication May 6, 1999.) 